\title{
INCORPORACIÓN DE ESPIRULINA (SPIRULINA MAXIMA) EN DIETAS PARA ALEVINES DE TRUCHAS ARCO IRIS (ONCORHYNCHUS MYKISS)
}

\section{INCORPORATION OF SPIRULINA (SPIRULINA MAXIMA) IN DIETS FOR RAINBOW TROUT (ONCORHYNCHUS MYKISS) FRY}

\author{
JOSÉ POKNIAK R. MV, MS.'
}

\begin{abstract}
A nutritional assay was conducted with rainbow trout fry (Oncorhynchus mykiss), from 2 up to $30 \mathrm{~g}$ body weight, using a spirulina meal (Spirulina maxima). The spirulina meal was incorporated in the feed at 0,$0 ; 2,5$ and $5,0 \%$ levels in order to verify the effects on productive performance, mortality (\%) and uric acid levels ( $\mathrm{mg} / \mathrm{dll}$ ). After 90 days, the productive performance (body weight, feed intake, feed conversion and specific growth rate) was not significantly affected $(P \geq 0,05)$ by the presence of spirulina meal in the diets. The mortality was not significantly $(P \geq 0,05)$ modified by the treatments, the same was observed in the uric acid levels. Under the experimental conditions used, the results obtained show that a $5 \%$ of spirulina meal can be incorporated in the feed for rainbow trout fry.
\end{abstract}

KEY WORDS: Spirulina; feeding; fry; rainbow trout.

PALABRAS Clave: Espirulina; alimentación; alevines; trucha arco iris.

\section{INTRODUCCIÓN}

La mayoría de las dietas que se emplean en los cultivos intensivos de salmónidos incluyen como fuente principal de proteína a la harina de pescado. En la actualidad se realizan esfuerzos por incorporar otros recursos que permitan disminuir su participación en las dietas de los peces de cultivo (Carter y Hauler, 2000; Glencross y col., 2006). Una posibilidad de reemplazo para la harina de pescado lo presenta la microalga espirulina (Spirulina maxima) que presenta un perfil de aminoácidos bastante aceptable (Cuadro 1), cuando se le compara con el de la harina de pescado. Al incorporar 2,5\% de harina de espirulina en dietas de salmón cereza (Oncorhynchus masou) mejoró el crecimiento de estos peces (Henson, 1990). Dallaire $y$ col. (2007) incluyeron harina de algas hasta $12,5 \%$ de la dieta sin efectos adversos para alevines de truchas arco iris (Oncorhynchus mykiss). La incorporación de espirulina a la dieta puede conllevar una limitante por su contenido de ácidos nucleicos,

Facultad de Ciencias Veterinarias y Pecuarias. Universidad de Chile. Departamento de Fomento de la Producción Animal.jpokniak@uchile.cl aunque se desconocen efectos nocivos en los peces relacionados con un exceso de estos compuestos en la dieta (De la Higuera y Cardenete, 1987). El propósito del trabajo fue evaluar la incorporación de diferentes porcentajes de harina de espirulina a la dieta de alevines de trucha arco iris.

\section{MATERIALES Y MÉTODOS}

Se utilizaron 1.080 truchas arco iris (O. mykiss), con un peso promedio inicial de 2,2 g y $4 \mathrm{~cm}$ de longitud, aproximadamente. Los peces se distribuyeron aletoriamente en grupos de 120 en 9 estanques de $0,2 \mathrm{~m}^{3}$ y un flujo de agua de $0,1 \mathrm{~L}^{\text {-seg }}$. Previo al inicio de la experiencia los peces se alimentaron con su dieta habitual durante una semana, para facilitar su adaptación a los estanques, los cuales fueron aseados diariamente. La experiencia se extendió por 90 días. El régimen de luz fue el natural y la concentración de oxígeno y temperatura del agua se registró semanalmente mediante un sensor electrónico. Se realizaron controles de peso, mediante balanza digital, al inicio, 30, 60 y 90 días de ensayo, de consumo de alimento semanal y de mortalidad diariamente. 
CUADRO 1

COMPOSICIÓN NUTRICIONAL DE LA ESPIRULINA

\begin{tabular}{l|r|l|r}
\hline Composición & $\%$ & Ácidos grasos & $m g^{-k g}$ \\
\hline Materia seca & 95,80 & Laurico & 204 \\
\hline Proteína & 64,20 & Palmítico & 18820 \\
\hline Extracto etéreo & 5,00 & Esteárico & 117 \\
\hline Fibra Cruda & 0,50 & Oleico & 2490 \\
\hline E.N.Nitrogenado & 19,50 & Linoleico & 12352 \\
\hline Ácidos nucleicos & 4,35 & Linolénico & 294 \\
\hline E. digestible calculada Mcal/kg & 2,84 & Linolénico & 10360 \\
\hline Aminoácidos & & Vitaminas & \\
\hline Histidina & 1,00 & Provitamina A & 1700 \\
\hline Lisina & 3,30 & Tiamina & 55 \\
\hline Metionina & 1,80 & Riboflavina & 40 \\
\hline Isoleucina & 3,70 & Piridoxina & 3 \\
\hline Arginina & 5,00 & Cianocobalamina & 2 \\
\hline Leucina & 5,60 & E & 190 \\
\hline Fenilalanina & 3,20 & Inositol & 350 \\
\hline Treonina & 3,50 & Ácido pantoténico & 11 \\
\hline Valina & 4,80 & Niacina & 118 \\
\hline Triptofano & 1,00 & Biotina & 0,4 \\
\hline Glicina & 3,10 & Ácido fólico & 0,5 \\
\hline Cistina & 0,60 & Pigmentos & \\
\hline Tirosina & 2,90 & Clorofila & 6800 \\
\hline & & Criptoxantina & 556 \\
\hline & & Echinona & 439 \\
\hline & & Zeaxantina & 316 \\
\hline & & \\
\hline & & & \\
\hline & & & \\
\hline
\end{tabular}

Las truchas fueron alimentadas manualmente, de lunes a sábado, seis veces al día hasta saciedad. Se emplearon tres tratamientos con tres repeticiones cada uno: $\mathrm{T}_{1}$ : control y los experimentales $\mathrm{T}_{2}$ y $\mathrm{T}_{3}$, que incluían: $0 ; 2,5$ y $5 \%$ de espirulina, respectivamente. Las dietas se presentan en el Cuadro 2. Dietas 1 y 2: para peces de 2 a $17 \mathrm{~g}$ y desde $17 \mathrm{a}$ $30 \mathrm{~g}$, respectivamente.

Se determinó la tasa de crecimiento específico $\left(\%\right.$ día $\left.^{-1}\right)=100 \times\left(\ln \left(\mathrm{W}_{2} / \mathrm{W}_{1}\right)\right) \times d-^{-1}$, donde $\mathrm{W}_{1} \mathrm{y}$ $\mathrm{W}_{2}$ son los pesos inicial y final, respectivamente, y $d$ el número de días. Al término del ensayo, bajo anestesia, se sacrificaron seis peces por repetición, a los cuales se les seccionó transversalmente la aleta caudal. La sangre se recolectó en tubos heparinizados para la determinación de ácido úrico empleando para dicho propósito la prueba colorimétrica por medio del Merckotest $\AA$. Los resultados se evaluaron por medio del análisis de la varianza; para las diferencias entre medias se recurrió a la prueba de Tukey. Las variables expresadas en porcentajes se transformaron previamente mediante el método de Bliss.

\section{RESULTADOS Y DISCUSIÓN}

La temperatura $\left(\mathrm{C}^{\circ}\right)$ y concentración de oxígeno del agua (ppm) alcanzaron valores promedios, para la experiencia, de 16,4 y 6,5, respectivamente. Estas condiciones ambientales pueden considerarse satisfactorias para el cultivo de truchas, aunque hubiese sido deseable una concentración de oxígeno más elevada, como lo indica Stevenson (1987); sin embargo, están dentro de lo recomendado por Sedwick (1983).

Los indicadores productivos se presentan en el Cuadro 3. Estos tuvieron un comportamiento muy semejante a lo largo y al término del ensayo en los tratamientos evaluados, sin mostrar diferencias sig- 
CUADRO 2

COMPOSICIÓN DE LAS DIETAS UTILIZADAS EN EL ENSAYO

\begin{tabular}{|c|c|c|c|c|c|c|}
\hline \multirow[b]{2}{*}{ Insumos $\%$} & \multicolumn{3}{|c|}{ Dietas 1} & \multicolumn{3}{|c|}{ Dietas 2} \\
\hline & $T 1$ & $T 2$ & $T 3$ & $T 1$ & $T 2$ & $T 3$ \\
\hline Trigo, harinilla & 14,81 & 13,06 & 12,02 & 23,86 & 24,55 & 27,20 \\
\hline Maravilla, afrecho & 9,18 & 7,85 & 3,76 & & & \\
\hline Pescado, harina & 68,89 & 65,25 & 66,09 & 45,88 & 43,94 & 43,80 \\
\hline Sangre, harina & 5,04 & 3,11 & & & & \\
\hline Carne, harina & 2,23 & 7,24 & 8,46 & 11,93 & 11,98 & 11,11 \\
\hline Espirulina, harina & 2,50 & 5,00 & 2,50 & 5,00 & & \\
\hline Pescado, aceite & 7,42 & 7,23 & 6,82 & 8,44 & 8,47 & 8,42 \\
\hline Vitaminas, p.m ${ }^{1}$ & 0,84 & 0,84 & 0,84 & 0,41 & 0,41 & 0,41 \\
\hline Minerales, p.m ${ }^{2}$ & 0,47 & 0,47 & 0,47 & & & \\
\hline Antifúngico & 0,11 & 0,11 & 0,11 & 0,11 & 0,11 & 0,11 \\
\hline Aglomerante & 0,19 & 0,19 & 0,19 & 0,19 & 0,19 & 0,19 \\
\hline \multicolumn{7}{|l|}{ Aportes } \\
\hline Proteína $^{3}$ & 61,50 & 59,30 & 60,35 & 49,70 & 48,80 & 49,97 \\
\hline Extracto etéreo $^{3}$ & 16,83 & 17,21 & 16,99 & 16,08 & 15,79 & 15,92 \\
\hline E. digestible calculada Mcal/kg & 4,35 & 4,30 & 4,25 & 3,89 & 3,87 & 3,85 \\
\hline
\end{tabular}

1 Premezcla vitamínica: g/kg p.m. A:12; D3:2; E: 100; K: 5; B2: 10; B12: 10; Ác. Pantoténico:25; Niacina: 75; Ác. Fólico: 2,5; Biotina: 50; Tiamina: 5; Piridoxina: 5; Inositol: 60.

2 Premezcla mineral: g/kg p.m. Calcio, carbonato: 86; Potasio, cloruro: 50; Cobre, sulfato: 0,97 Manganeso, óxido: 1,4; Magnesio, óxido: 42,4; Cobalto, óxido: 0,232; Sodio, selenito: 0,014; Fierro, sulfato: 25; Zinc, sulfato: 5,51; Calcio. Yodato: 2,84.

3 Determinada por análisis químico proximal.

\section{CUADRO 3 \\ PESO VIVO, CONSUMO DE ALIMENTO, CONVERSIÓN ALIMENTICIA (CONSUMO/GANANCIA PESO), TASA DE CRECIMIENTO ESPECÍFICO (TCE) Y NIVELES DE ÁCIDO ÚRICO DE TRUCHAS ARCO IRIS. PROMEDIOS \pm DESVIACIÓN ESTÁNDAR}

\begin{tabular}{lc|c|c}
\hline & \multicolumn{3}{c}{ Tratamientos } \\
\cline { 2 - 4 } Variables & 1 & 2 & 3 \\
\hline Peso vivo $(\mathrm{g})$ & $2,23 \pm 0,08$ & $2,17 \pm 0,29$ & $2,11 \pm 0,21$ \\
\hline Inicial & $32,26 \pm 5,08$ & $31,26 \pm 5,56$ & $27,52 \pm 2,15$ \\
\hline Final & $23,33 \pm 1,91$ & $23,52 \pm 2,14$ & $22,52 \pm 0,40$ \\
\hline Consumo $(\mathrm{g})$ & $0,79 \pm 0,08$ & $0,82 \pm 0,10$ & $0,89 \pm 0,06$ \\
\hline Conversión & $3,01 \pm 0,21$ & $3,00 \pm 0,34$ & $2,90 \pm 0,10$ \\
\hline TCE \% & $0,09 \pm 0,03$ & $0,07 \pm 0,02$ & $0,10 \pm 0,04$ \\
\hline Ácido úrico $\mathrm{mg}^{\text {-dl }}$ & &
\end{tabular}

nificativas $(P \geq 0,05)$ entre ellos en ninguno de los indicadores estudiados. El consumo se corrigió por la mortalidad de cada mes.

El peso promedio final se ajustó a las tablas de predicción consultadas que consideraban 90 días para alcanzar un peso de $30 \mathrm{~g}$, como también con Pérez (1991), para truchas provenientes de ovas importadas y nacionales durante el mismo periodo de crecimiento. Aunque no hubo diferencias significativas entre los tratamientos para este indicador, a medida que se incrementó la participación de la harina de espirulina en las dietas el peso tendió a declinar, lo que podría atribuirse a una menor digestibilidad de las dietas que incluían a las microalgas $\left(\mathrm{T}_{2} \mathrm{y} \mathrm{T}_{3}\right)$ en 
comparación a la harina de pescado, como lo señalan De la Higuera y Cardenete (1987).

Respecto del consumo de alimento podría esperarse el comportamiento observado, prácticamente igual entre los tratamientos, puesto que las dietas se formularon isoenergéticas, y siendo la energía un factor clave en la regulación del consumo de los peces (Cho y Kaushik, 1990; Guillaume y col., 2001), los resultados logrados confirman esta situación. Por otra parte, las variables ambientales fueron las apropiadas para el cultivo de las truchas, no influyendo sobre este indicador. Lo que se debería destacar es que la incorporación de harina de espirulina hasta un 5\% de la dieta no alteró la aceptabilidad de éstas, factor que podría haber modificado el valor de este parámetro.

La conversión de alimentos mostró una respuesta semejante a la informada por Boonyaratpalin y Unprasert (1989) cuando incorporaron 10\% de harina de espirulina a la dieta de tilapia roja (Oreochromis niloticus) y fueron coincidentes con lo reportado por Pérez (1991) para truchas en etapas iniciales de crecimiento. Los valores de conversión inferiores a la unidad son comunes en peces por las razones expuestas por Lovell (1989).

La TCE fue decreciendo a lo largo de la experiencia, los valores que se entregan representan los promedios generales para los diferentes tratamientos que fueron coincidentes a los reportados por Pérez (1991) para truchas durante un periodo de crianza igual al de este ensayo. En la medida que los peces aumentan de peso la TCE se deteriora por el incremento de su gasto de mantención (Guillaume y col., 2001).

Los niveles de ácido úrico circulante (Cuadro 3) no pusieron en evidencia el posible efecto de la incorporación de harina de espirulina a las dietas, lo que conllevaba una sobrecarga de ácidos nucleicos para las truchas y que podían constituirse en una limitante en el uso de este recurso. Los resultados demostraron la capacidad que tienen los peces para transformar el ácido úrico, producto residual del metabolismo de los ácidos nucleicos, en urea (Cowey, 1988).

La mortalidad acumulada para todo el ensayo fue de 0,$70 ; 1,10$ y $1,39 \%(\mathrm{P} \geq 0,05)$ para los tratamientos 1,2 y 3 , respectivamente; porcentajes que están por bajo el 3\% informado por Pérez (1991) para trucha durante esta etapa de crianza. Boonyaratpalin y Unprasert (1989) tampoco observaron un aumento en la mortalidad cuando se incorporó un $10 \%$ de harina de espirulina a la dieta de tilapia roja (Oreochromis niloticus), incluso Dallaire y col. (2007) no observaron mortalidad durante 10 semanas al incluir harina de algas hasta en un $50 \%$ de la dieta de alevines de trucha arco iris. Lo anterior sugiere que existe un margen de seguridad amplio en cuanto al nivel de incorporación de harina de algas a la dieta de truchas arco iris, que no afectará negativamente la sobrevida y la respuesta productiva de los peces. Al finalizar el ensayo, se observó una mayor intensidad en la pigmentación de la piel en las truchas alimentadas con $5,0 \%$ de espirulina.

En definitiva, la incorporación de harina de espirulina a la dieta de truchas arco iris y de salmones dependerá tanto de su disponibilidad como de su precio.

\section{RESUMEN}

Se realizó un ensayo con alevines de trucha arco iris (Oncorhynchus mykiss), desde los 2 hasta los $30 \mathrm{~g}$ de peso, usando harina de espirulina (Spirulina maxima) en niveles de $0 ; 2,5$ y $5 \%$ de la dieta. Se evaluó la respuesta productiva (peso vivo, consumo de alimento, conversión de alimento y tasa de crecimiento específico), mortalidad (\%) y ácido úrico circulante $\left(\mathrm{mg}^{-\mathrm{dl}}\right)$. Después de 90 días de ensayo, los indicadores productivos no fueron modificados significativamente $(\mathrm{P} \geq 0,05)$ por la presencia de la harina de espirulina en las dietas, como tampoco lo fueron la mortalidad y el ácido úrico circulante. Bajo las condiciones experimentales empleadas, los resultados mostraron que un $5 \%$ de la harina de espirulina se puede incluir en la dieta para alevines de trucha arco iris.

\section{REFERENCIAS}

BOONYARATPALIN, N.; UNPRASERT, N. 1989. Effects of pigments from different sources on colour changes and growth of red tilapia (Oreochromis niloticus). Aquaculture 79:375-380.

CARTER, C.G.; HAULER, R.C. 2000. Fish meal replacement by plant meals in extruded feeds for Atlantic salmon, Salmo salar L. Aquaculture 185:299-311.

CHO, C; KAUSHIK, S. 1990. Nutricional energetic in fish: energy and protein utilization in rainbow trout (Salmo gairdneri). World Review of Nutrition and Dietetics. 61: 132:172.

COWEY, C. 1988. The nutrition of fish: the developing scene. Nutrition Reviews 1:255-280.

Dallaire, V; Lessard, P; VAndenberG, G; DE la NoüE, J. 2007. Effect of algal incorporation on growth, survival and carcass composition of rainbow trout (Oncorhynchus mykiss) fry. Bioresource Technology 98 (7):1433-1439. 
De la Higuera, M.; Cardenete, G. 1987. Fuentes alternativas de proteína y energía en acuicultura. Alimentación en Acuicultura. Comisión Asesora de Investigación Científica y Técnica (CAICYT). Madrid, España. Industria Gráfica, S.L. 325 pp.

GLENCROSS, B; HAWKINS, W; EVANS, D; RUTHERFORD, N; DODS, K; MAAS, R; MCCAFFERTY, P; SIPSAS, S. 2006. Evaluation of the nutritional value of prototype lupin protein concentrates when fed to rainbow trout (Oncorhynchus mykiss). Aquaculture 251:66-77.

Guillaume, J; Kaushik, S; Bergot, P; Métailler, R. 2001. Nutrition and Feeding of fish and crustaceans. Praxis Publishing Ltd, Chichester, UK. 408 p.
HENSON, R. 1990. Spirulina algae improves Japanese fish feeds. Aquaculture Magazine. Nov/Dec. Pp. 38-43.

LOVELL, T. 1998. Nutrition and Feeding of fish. 2nd. Ed. Kluwer Academic Publishers. 267 pp.

PÉREZ, P. 1991. Comparación del comportamiento productivo de truchas arco iris (Oncorhynchus mykiss) provenientes de ovas importadas y nacionales. Memoria de Título. Santiago. Universidad de Chile. Facultad de Ciencias Veterinarias y Pecuarias. $60 \mathrm{pp}$.

SEDWICK, D.S. 1983. Trout farming handbook. England. Fishing New Books. 160 pp.

StEVENSON, J.P. 1987. Trout farming manual. 2nd. Ed. England. Finishing New Book. 259 pp. 